\title{
Directed packings of circles in the plane
}

\author{
Tomislav Došlić \\ University of Zagreb, Faculty of Civil Engineering, \\ Kačićeva 26, 10000 Zagreb, Croatia \\ Mirjam Škegro \\ Erste \& Steiermärkische Bank d.d., \\ Ivana Lučića 2, 10000 Zagreb, Croatia
}

\begin{abstract}
We consider sequential packings of families of circles in the plane whose curvatures are given as members of a sequence of non-negative real numbers. Each such packing gives rise to a sequence of circle centers that might diverge to infinity or remain bounded. We examine the behavior of the sequence of circle centers as a function of the growth rate of the sequence of curvatures. In several special cases we obtain explicit formulas for the coordinates of the limit, while in other cases we obtain accurate estimates.
\end{abstract}

Keywords: packing of circles

MSC: 05B40, 51M04, 52C15

\section{Introduction and motivation}

Packings of geometric objects have been attracting attention of researchers and engineers since the antiquity [1]. Probably the best known example is the celebrated Kepler conjecture on sphere packings in space, centuries old, and solved only recently

DOI: https://doi.org/10.5592/CO/CCD.2018.03 
$[2,3]$. Many other problems of efficient use of (not necessarily spatial) resources can be successfully modeled by packings. Among the examples are accretion processes of impenetrable particles subject to attractive forces. A toy model of one such process is considered in this paper. We construct it by considering dynamic (sequential) packing of circles in the plane subject to the following conditions.

In the beginning (at the time zero), we have two circles touching externally at the origin of the plane coordinate system. Both their centers are on the $y$-axis, and we allow that one of the circles has an infinitely large radius (hence the curvature equal to zero). At each successive moment a circle arrives and settles in the plane so that it touches externally two circles that immediately precede it. Out of the two possible location for the circle center, we choose the one that is farther from the origin. We are interested in the behavior of the sequence of centers of circles in such packings. More precisely, we wish to decide whether the sequence of centers diverges to infinity or converges to a limit, i.e., to a point at a finite distance from the origin. In the latter case, we would like also to determine the coordinates of the limit, or, at least, to give some estimate of its location.

It is clear that the behavior of the sequence of circle centers depends on the radii of accreting circles. We assume that the inverse values of the radii (hence the circle curvatures) are given as elements of a sequence $\left(a_{n}\right)_{n \geqslant 0}$ of non-negative real numbers. Our goal is to determine how behavior of the sequence $\left(S_{n}\right)_{n \geqslant 0}$ of circle centers depends on the sequence $\left(a_{n}\right)_{n \geqslant 0}$. In particular, we aim at finding the necessary and sufficient conditions for convergence of $\left(S_{n}\right)_{n \geqslant 0}$ in terms of growth properties of sequence of curvatures. In case of convergence, we will try to determine the exact or approximate coordinates of the limit.

\section{Definitions and preliminaries}

Let $I$ be an arbitrary index set. A family $\left\{K_{i}, i \in I\right\}$ of sets in the plane is a packing if $i n t K_{i} \cap i n t K_{j} \neq \emptyset, \forall i \neq j \in I$.

In our case, $I=\mathbb{N}_{0}$ and each $K_{i}$ is a circle of radius $r_{i}$ centered at $S_{i}$. It is clear that 
the efficiency of packing will be the greatest if the circles touch each other (since their interiors cannot overlap).

Let us take a sequence $\left(a_{n}\right)_{n \geqslant 0}$ of non-negative real numbers such that $a_{n}>0$ for all $n \geqslant 1$ and two circles, $K_{0}$ and $K_{1}$ so that they touch externally at the origin of the plane coordinate system. We assume that the center of $K_{0}$ is on the negative part of the $y$-axis and the center of $K_{1}$ is on the positive part of the $y$-axis. We denote these centers by $S_{0}$ and $S_{1}$, respectively. The radii $r_{0}$ and $r_{1}$ of $K_{0}$ and $K_{1}$ are given as the reciprocal values of $a_{0}$ and $a_{1}$, respectively. Clearly, if $a_{0}=0, K_{0}$ becomes the $x$-axis; such a situation is shown in Fig. 1 . Now take a circle $K_{2}$ of

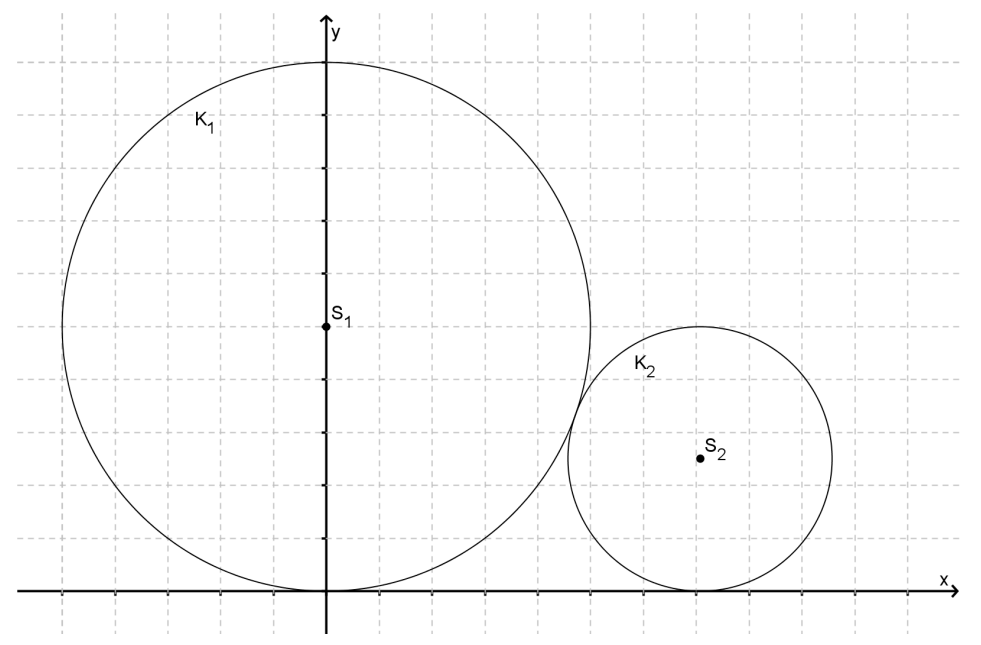

Figure 1: The case $a_{0}=0$

radius $r_{2}=\frac{1}{a_{2}}$ and position it in the right half-plane so that it touches externally both $K_{0}$ and $K_{1}$. Denote its center by $S_{2}$. Now, for each $n \geqslant 3$ take a circle $K_{n}$ of radius $r_{n}=\frac{1}{a_{n}}$ and determine its center $S_{n}$ so that $K_{n}$ touches externally both $K_{n-1}$ and $K_{n-2}$. In each step there will be two possible locations of $S_{n}$; we take the one more distant from the origin. In the rest of this paper we will study how the behavior of so constructed sequence $\left(S_{n}\right)_{n \geqslant 0}$ depends on properties of $\left(a_{n}\right)_{n \geqslant 0}$.

Let $S\left(\left(a_{n}\right)\right)$ denote the limit (if it exists) of the sequence of circle centers for a given sequence $\left(a_{n}\right)$, and let $\rho\left(\left(a_{n}\right)\right)$ denotes its distance from the origin. When $\left(S_{n}\right)$ diverges, we set $\rho\left(\left(a_{n}\right)\right)=\infty$. Our problem can be now formulated in terms 
of $\rho\left(\left(a_{n}\right)\right)$ : whether it is finite or infinite for a given $\left(a_{n}\right)$, and, if finite, what is its exact or approximate value.

We start by some simple results for the case of constant and of decreasing sequences $\left(a_{n}\right)_{n \geqslant 0}$. In the case of constant sequence we can assume $a_{n}=1$ for all $n \geqslant 0$. The situation is shown in Fig. 2. It is a simple exercise to compute the coordinates $\left(p_{n}, q_{n}\right)$ of the sequence of centers $S_{n}[4]$.

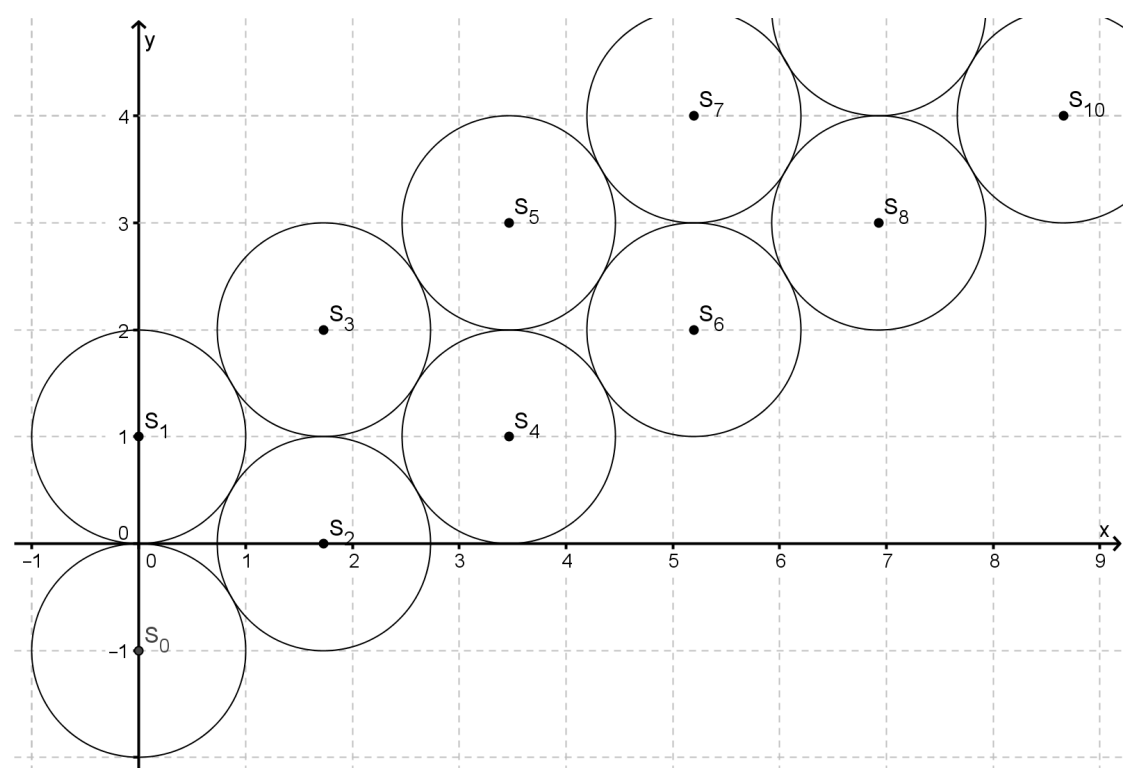

Figure 2: The packing for $a_{n}=1$

$$
p_{n}=\left\lfloor\frac{n}{2}\right\rfloor \sqrt{3}, \quad q_{n}=3\left\lfloor\frac{n+1}{2}\right\rfloor-n-1 .
$$

It is clear from both the figure and from the explicit expression for the coordinates of $S_{n}$ that the sequence $\left(S_{n}\right)$ diverges.

Another simple situation arises when the sequence $\left(a_{n}\right)_{n \geqslant 0}$ is decreasing. An example of such a situation with $a_{0}=0, a_{n}=\frac{1}{n}$ for $n \geqslant 1$, is shown in Fig. 3 . It is clear that the sequence $\left(S_{n}\right)$ diverges. Hence, it makes sense to restrict our attention on 


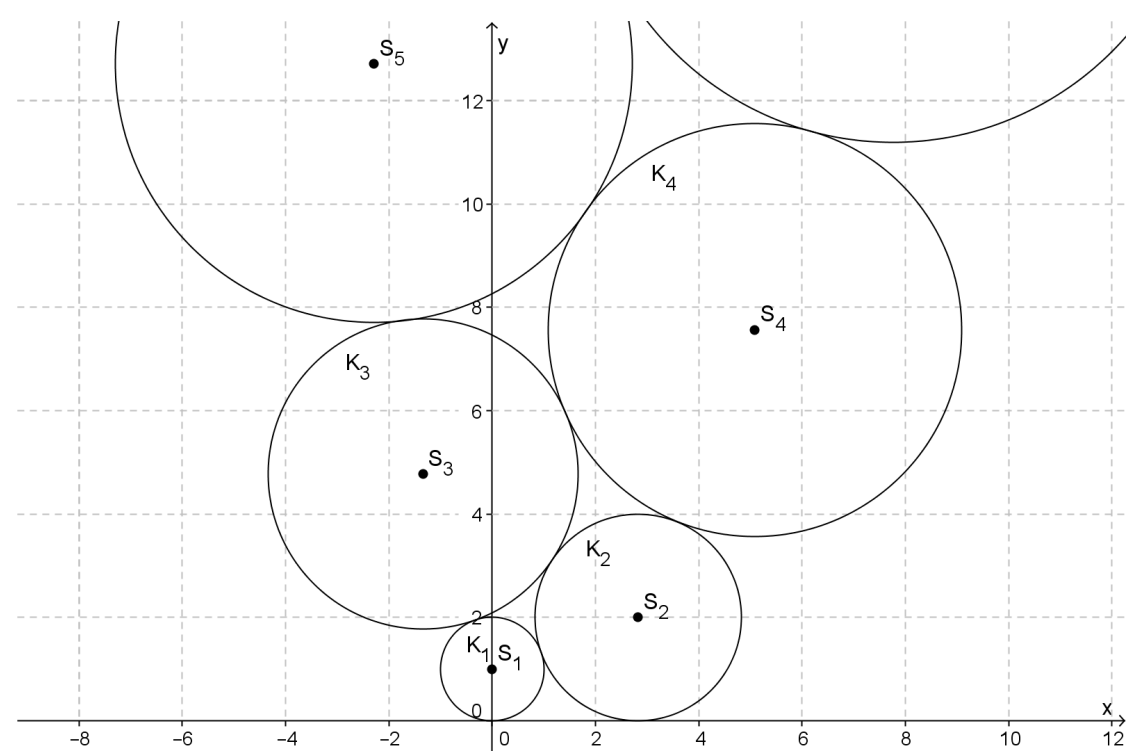

Figure 3: A packing with an inadmissible sequence $\left(a_{n}\right)$

(at least weakly) increasing sequences that grow without bound. From now on we consider only such sequences. We call them the admissible sequences.

By using induction on $n$ and passing to the limit we can establish the following property of admissible sequences.

Proposition 1 Let $\left(a_{n}\right)_{n \geqslant 0}$ and $\left(b_{n}\right)_{n \geqslant 0}$ be two admissible sequences with $a_{n} \leqslant b_{n}$ for all $n \in \mathbb{N}_{0}$. Then $\rho\left(\left(a_{n}\right)\right) \geqslant \rho\left(\left(b_{n}\right)\right)$.

We close this section by considering the case when the sequence of radii converges, hence when $\sum_{n=0}^{\infty} \frac{1}{a_{n}}=M<\infty$.

Proposition 2 If the series $\sum \frac{1}{a_{n}}$ is convergent, then the sequence $\left(S_{n}\right)$ converges to the limit $S\left(\left(a_{n}\right)\right)$ at a finite distance from the origin. 
Proof. Let us denote by $d_{n}$ the distance between $S_{n}$ and the origin. It is clear that the difference $d_{n}-d_{n-1}$ cannot exceed the sum of the radii of the corresponding circles. Hence, $d_{n}-d_{n-1} \leqslant \frac{1}{a_{n-1}}+\frac{1}{a_{n}}$ for all $n \geqslant 2$. The claim now follows by summing over all $n \geqslant 2$.

Corollary 3 Let $\left(a_{n}\right)_{n \geqslant 0}$ be an admissible sequence and let $a_{n} \in \Omega\left(n^{s}\right)$ for some $s>1$. Then $\rho\left(\left(a_{n}\right)\right)<\infty$.

The above result settles the case of sequences $\left(a_{n}\right)$ growing faster than the sequence of natural numbers. It leaves open the case when $\left(a_{n}\right)$ is exactly the sequence of natural numbers, as well as all admissible sequences of slower growth. Also, it provides no information on the actual values of $S\left(\left(a_{n}\right)\right)$ and $\rho\left(\left(a_{n}\right)\right)$ for convergent sequences $\left(S_{n}\right)$.

\section{$3 \quad$ Numerical experiments}

In order to facilitate our investigation and to gather some information on behavior of various sequences, we have designed a recursive algorithm for computing the coordinates of centers $S_{n}$ for a given sequence $\left(a_{n}\right)$. It is based on the procedure for finding intersections of two circles with given centers and radii. We have implemented the algorithm in Matlab; the code is given in the Appendix.

\subsection{Algorithm}

We start from a given sequence $\left(a_{n}\right)_{n \geqslant 0}$, and the sequence of reciprocal values $\left(r_{n}\right)_{n \geqslant 0}=\left(\frac{1}{a_{n}}\right)_{n \geqslant 0}$. We take circles $K_{0}$ and $K_{1}$ centered at $S_{0}=\left(0,-\frac{1}{a_{0}}\right)$ and $S_{1}=\left(0, \frac{1}{a_{1}}\right)$, respectively, touching externally at the origin. For $n \geqslant 2$ we solve the system

$$
\left\{\begin{array}{l}
\left(x-p_{n-2}\right)^{2}+\left(y-q_{n-2}\right)^{2}=\left(r_{n-2}+r_{n}\right)^{2} \\
\left(x-p_{n-1}\right)^{2}+\left(y-q_{n-1}\right)^{2}=\left(r_{n-1}+r_{n}\right)^{2}
\end{array}\right.
$$


for $p_{n}$ and $q_{n}$, the coordinates of $S_{n}$. The solutions represent two intersection of circles centered at $S_{n-2}$ and $S_{n-1}$ with radii $r_{n-2}+r_{n}$ and $r_{n-1}+r_{n}$, respectively, and at each step we choose the one farther from the origin. The first step is shown in Fig. 4. In case of $a_{0}=0$, the first step is modified so as to acknowledge the fact

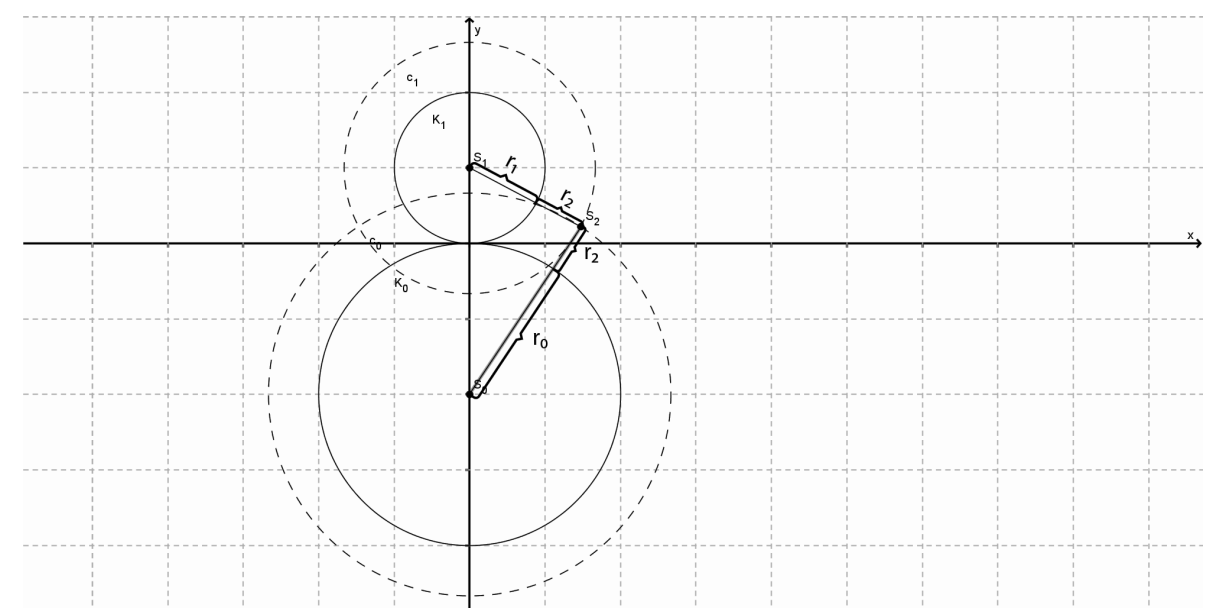

Figure 4: Start of the algorithm.

that one circle is actually a line, the $x$-axis. The modification is shown in Fig. 5.

For $n \geqslant 2$ (or $n \geqslant 3$ if $a_{0}=0$ ) we define

$$
\begin{aligned}
& k_{n-2}:=\frac{p_{n-2}-p_{n-1}}{q_{n-1}-q_{n-2}} \\
& l_{n-2}:=\frac{r_{n-2}^{2}-r_{n-1}^{2}+\left(2 r_{n-2}-2 r_{n-1}\right) r_{n}-p_{n-2}^{2}+p_{n-1}^{2}-q_{n-2}^{2}+q_{n-1}^{2}}{2 q_{n-1}-2 q_{n-2}} . \\
& a_{n-2}^{\prime}=1+k_{n-2}^{2} \\
& b_{n-2}=2 k_{n-2} l_{n-2}-2 p_{n-2}-2 q_{n-2} k_{n} \\
& c_{n-2}=-r_{n-2}^{2}-2 r_{n-2} r_{n}-r_{n}^{2}+p_{n-2}^{2}-2 q_{n-2} l_{n}+q_{n-2}^{2}+l_{n}^{2} .
\end{aligned}
$$

After some tedious, but otherwise quite straightforward, algebraic manipulations, we obtain a recursive formula for computing sequences $\left(p_{n}\right)$ and $\left(q_{n}\right)$ of center co- 


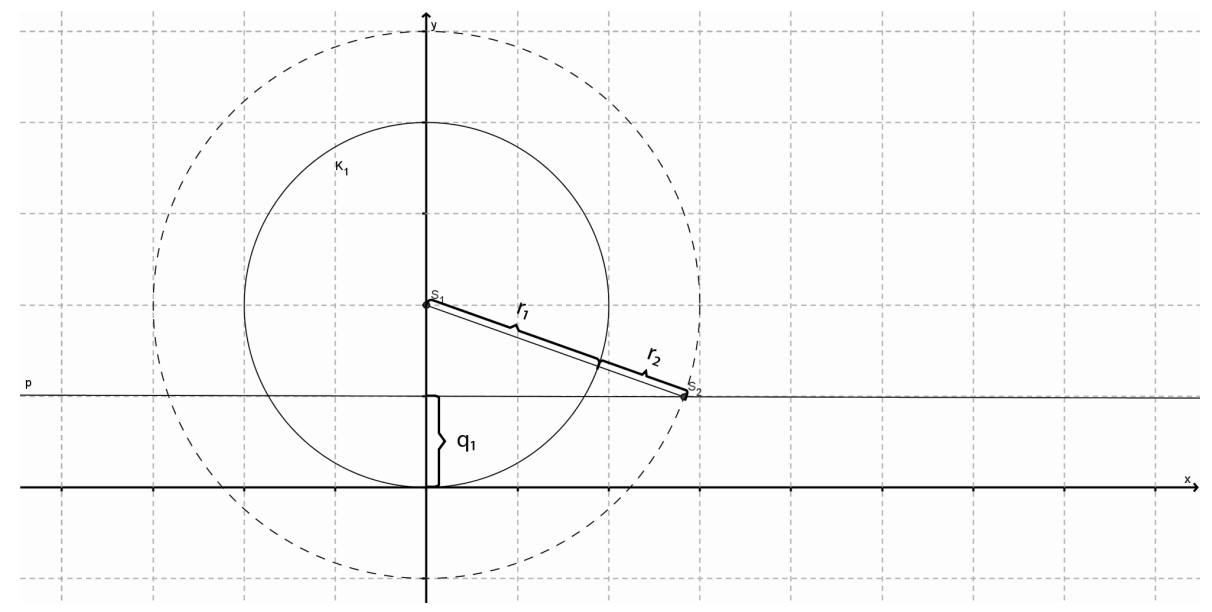

Figure 5: Start of the algorithm for $a_{0}=0$.

ordinates.

$$
\begin{aligned}
& p_{n}=\frac{-b_{n-2}+\sqrt{b_{n-2}^{2}-4 a_{n-2}^{\prime} c_{n-2}}}{2 a_{n-2}^{\prime}}, \\
& q_{n}=k_{n} p_{n}+l_{n} .
\end{aligned}
$$

In the next subsection we show the results of the algorithm for two sequences with convergent sequences of centers.

\subsection{Examples}

Our first example is representative for all geometric sequences $q^{n}$ for $q>1$.

Example $4 a_{n}=2^{n}$.

The values for the first ten iterations are shown in Table 1. 


\begin{tabular}{|c||c|c|c|}
\hline$n$ & $p_{n}$ & $q_{n}$ & $r_{n}$ \\
\hline 0 & 0 & -1 & 1 \\
\hline 1 & 0 & 0.5 & 0.5 \\
\hline 2 & 0.62361 & 0.083333 & 0.25 \\
\hline 3 & 0.62361 & 0.458333 & 0.125 \\
\hline 4 & 0.779512 & 0.354167 & 0.0625 \\
\hline 5 & 0.779512 & 0.447917 & 0.03125 \\
\hline 6 & 0.818488 & 0.421875 & 0.015625 \\
\hline 7 & 0.818488 & 0.445312 & 0.007813 \\
\hline 8 & 0.828231 & 0.438802 & 0.003906 \\
\hline 9 & 0.828231 & 0.444661 & 0.001953 \\
\hline 10 & 0.830667 & 0.443034 & 0.000977 \\
\hline$\ldots$ & $\ldots$ & $\ldots$ & $\ldots$ \\
\hline
\end{tabular}

We observe that $p_{2 k}=p_{2 k+1}$ for $k \geqslant 0$; the pattern will be rigorously established later. By increasing the number of iterations and zooming the corresponding pictures we can obtain approximate coordinates of $S\left(\left(2^{n}\right)\right)$. The process is illustrated in Figures 6 and 7 .

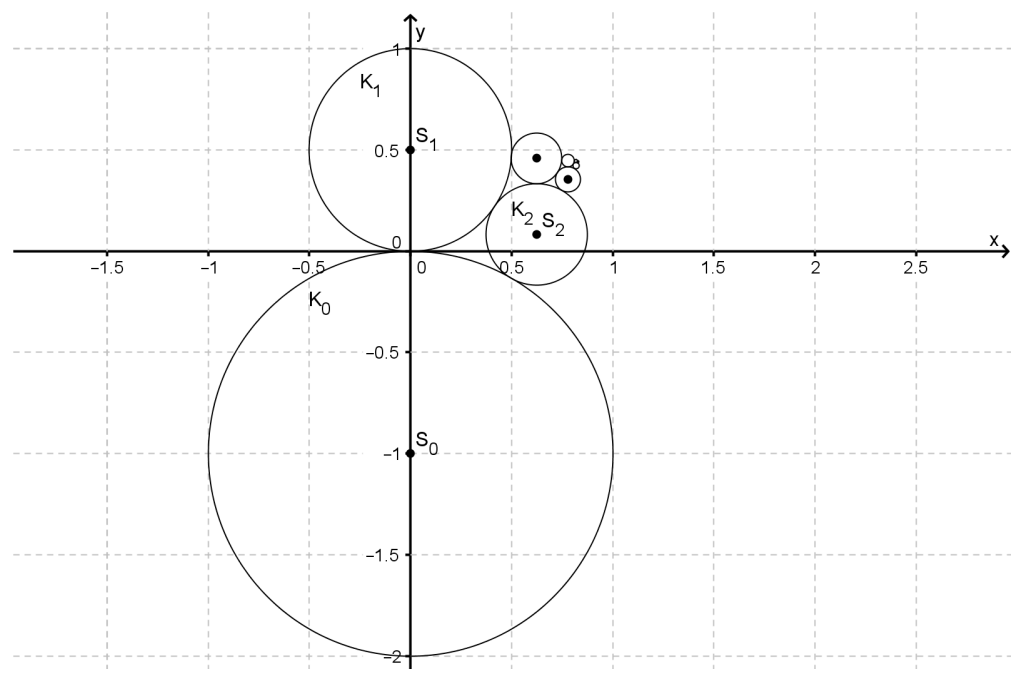

Figure 6: $a_{n}=2^{n}$ 


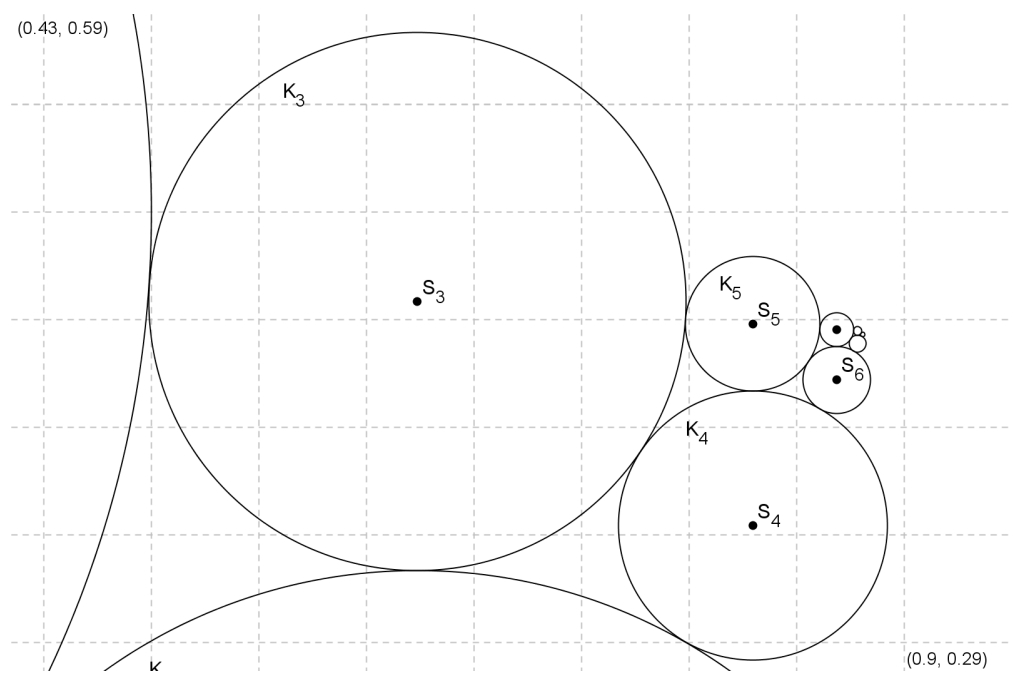

Figure 7: Zoom for $a_{n}=2^{n}$

Example $5 a_{n}=n^{2}$.

Here we omit the table and show only graphical representation (see Figure 8).

\section{Exact approach}

In this section we first introduce and study the mesh of triangles defined by the circle centers. The mesh is shown in Fig. 9. It is fully enclosed between two piecewise linear curves made of segments connecting the centers of successive even- and oddnumbered circles in the packing. For a given admissible sequence $\left(a_{n}\right)$, let us denote by $T_{n}$ the triangle with vertices $S_{n-1}, S_{n}, S_{n+1}$ for $n \geqslant 1$ if $a_{0} \neq 0$ and for $n \geqslant 2$ if $a_{0}=0$. Its sides have lengths $\frac{1}{a_{n}}+\frac{1}{a_{n+1}}, \frac{1}{a_{n-1}}+\frac{1}{a_{n+1}}$, and $\frac{1}{a_{n}}+\frac{1}{a_{n-1}}$. By using Heron's formula we obtain the area of $T_{n}$ as

$$
P_{n}=\frac{\sqrt{a_{n} a_{n-1}+a_{n-1} a_{n+1}+a_{n} a_{n+1}}}{a_{n-1} a_{n} a_{n+1}} .
$$




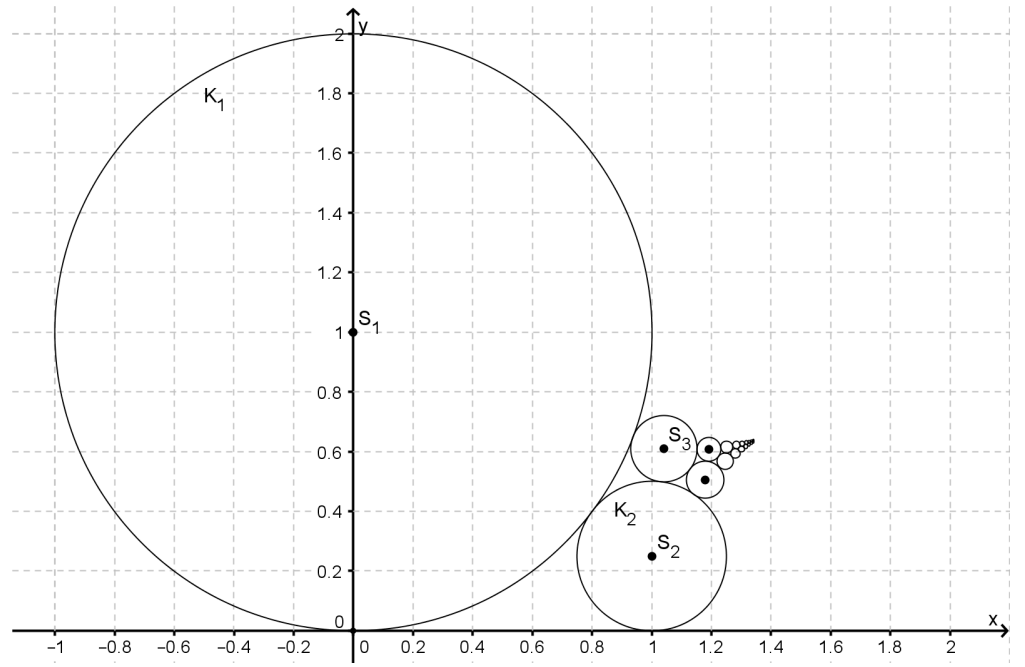

Figure 8: $a_{n}=n^{2}$

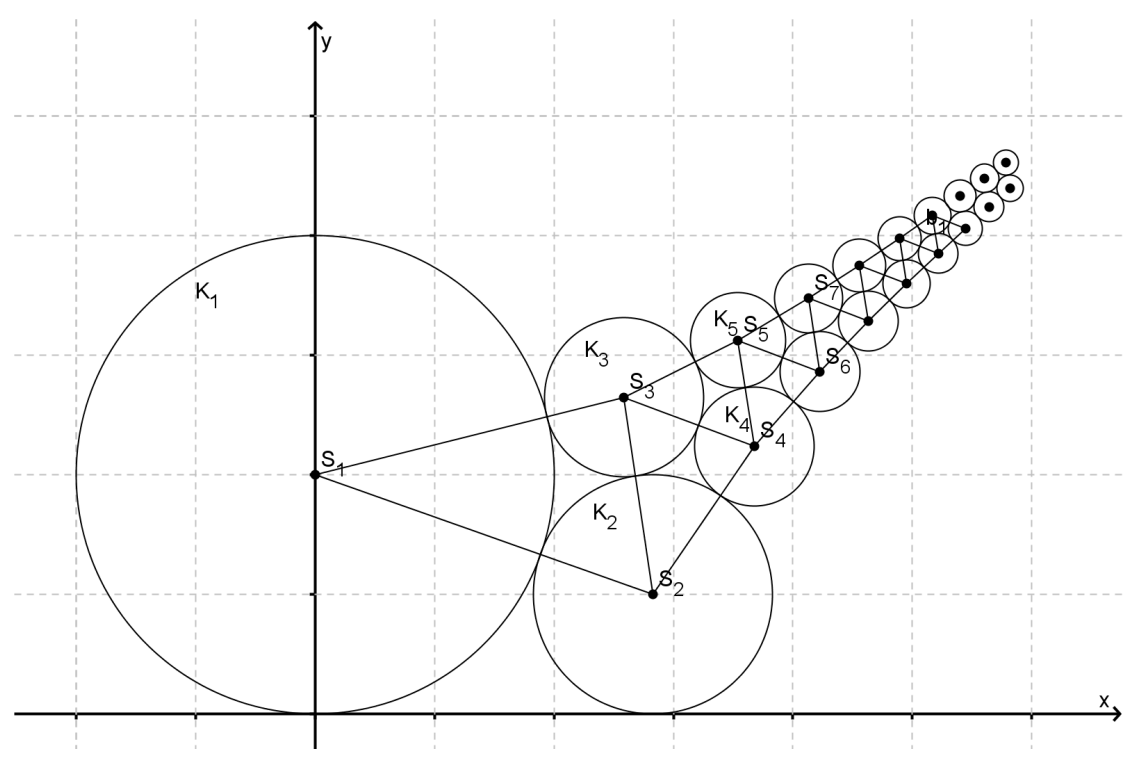

Figure 9: Triangle mesh. 
Now we look at the sum of all triangle areas. If the series $\sum_{n=1}^{\infty} P_{n}$ diverges, then the sequence $S_{n}$ must also diverge, since an infinite area cannot be enclosed by a circle of a finite radius. This immediately yields a lower bound for the growth rate of sequences with convergent center sequences.

Proposition 6 Let $\left(a_{n}\right)$ be an admissible sequence and $a_{n} \in O(\sqrt{n})$. Then $\rho\left(\left(a_{n}\right)\right)=\infty$.

Proof. $a_{n} \in O(\sqrt{n})$ means that there is $C>0$ such that $a_{n} \leqslant C \sqrt{n}, \forall n$. It is easy to see that for the sequence $b_{n}=C \sqrt{n}$ the corresponding sequence $P_{n}$ diverges. Hence $\rho\left(\left(b_{n}\right)\right)=\infty$, and then, by Proposition 1, also $\rho\left(\left(a_{n}\right)\right)=\infty$.

We notice that convergence of $\sum_{n=1}^{\infty} P_{n}$ does not imply the convergence of the sequence of circle centers, as a finite area can be enclosed between two curves of infinite length. Similarly, the divergence of the sum of distances between the centers of successive circles does not imply the divergence of the sequence of centers, since a curve of infinite length can be enclosed within a circle of a finite diameter. A nice example is provided by the Koch curve.

\section{1 $\quad a_{n}=n$}

In this subsection we consider and settle the case $a_{n}=n$. The mesh of triangles is shown in Fig. 10. It is easy to see that both $\left|\overline{S_{2} S_{4}}\right|+\left|\overline{S_{4} S_{6}}\right|+\left|\overline{S_{6} S_{8}}\right|+\ldots=\frac{1}{2}+\sum_{k=2}^{\infty} \frac{1}{k}$ and $\left|\overline{S_{1} S_{3}}\right|+\left|\overline{S_{3} S_{5}}\right|+\left|\overline{S_{5} S_{7}}\right|+\ldots=1+2 \cdot \sum_{k=1}^{\infty} \frac{1}{2 k+1}$ diverge. It does not mean, however, that the sequence of centers diverges. The divergence will follow only if we show that the piecewise linear curves bounding our mesh do not vary wildly, i.e., that they have certain monotonicity properties. In order to establish that monotonicity, look at Fig. 11 and consider angles $\alpha_{1}, \alpha_{2}, \alpha_{3}$. We would like to establish a lower bound on the sum of those angles $\alpha_{1}+\alpha_{2}+\alpha_{3}$. If that lower bound exceeds $\pi$, it means that the slopes of successive segments are non-increasing and remain positive. That will further imply that the piecewise linear curve is a graph of a function and hence cannot achieve its infinite length over a finite segment. 


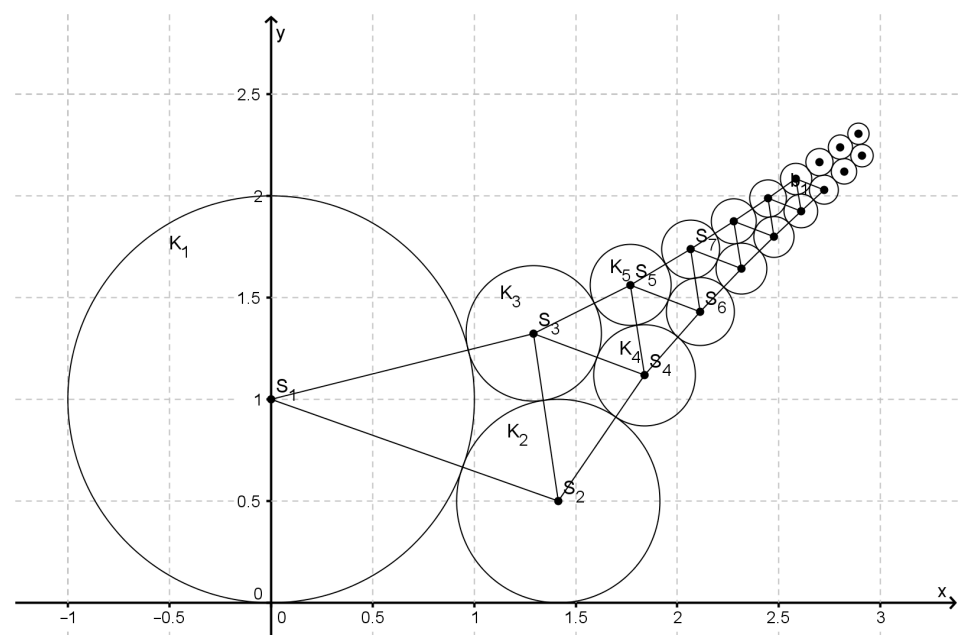

Figure 10: Triangle mesh for $a_{n}=n$

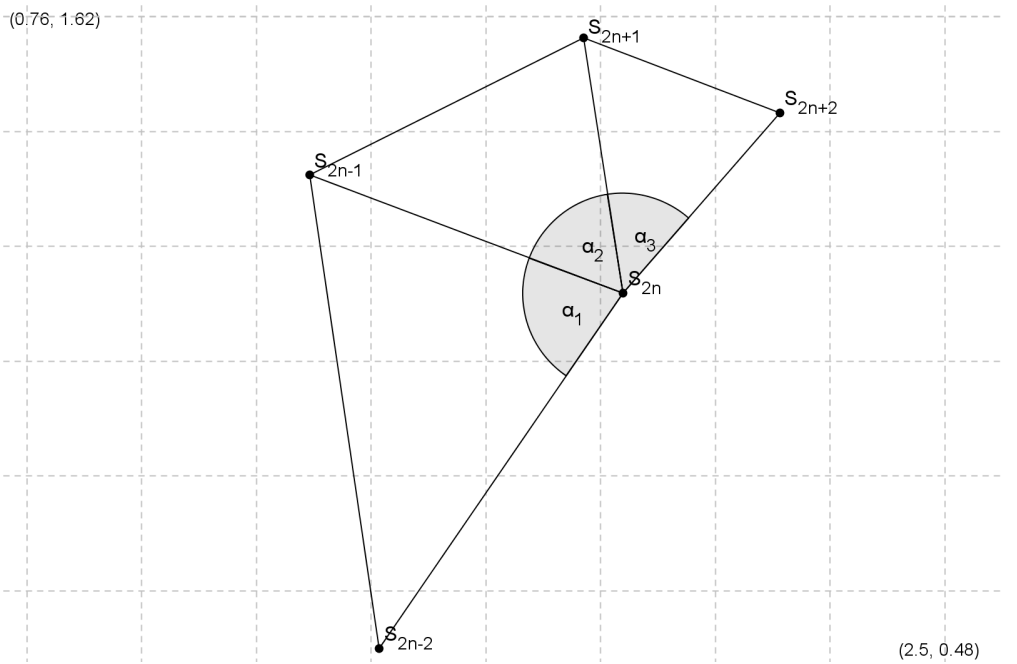

Figure 11: Angles at the center of an even-numbered circle for $a_{n}=n$

Consider the triangle $S_{2 n-2} S_{2 n-1} S_{2 n}$. By Heron's formula, its area is given by

$$
P_{2 n-1}=\frac{\sqrt{12 n^{2}-12 n+2}}{2 n(2 n-1)(2 n-2)} .
$$


From that, by formula $P=\frac{a b \sin \gamma}{2}$, we obtain

$$
\sin \alpha_{1}=\frac{4 n \sqrt{12 n^{2}-12 n+2}}{16 n^{2}-12 n+2} .
$$

Consider now the function $f(n):=\frac{4 n \sqrt{12 n^{2}-12 n+2}}{16 n^{2}-12 n+2}$. It tends toward $\frac{\sqrt{3}}{2}$ as $n \rightarrow \infty$. By taking derivative with respect to $n$ it is easy to see that $f$ is decreasing for $n>$ $\frac{5+\sqrt{13}}{4}$. Hence, $f(n)$ is approaching the value $\frac{\sqrt{3}}{2}$ from above for $n>\frac{5+\sqrt{13}}{4} \approx 2.15$. From there it follows $\sin \alpha_{1}>\frac{\sqrt{3}}{2}$ and hence $\alpha_{1}>\frac{\pi}{3}$.

In a similar way, by considering the triangle $S_{2 n-1} S_{2 n} S_{2 n+1}$ with area

$$
P_{2 n}=\frac{\sqrt{12 n^{2}-1}}{2 n(2 n-1)(2 n+1)},
$$

we obtain

$$
\sin \alpha_{2}=\frac{4 n \sqrt{12 n^{2}-1}}{16 n^{2}-1} .
$$

The function $g(n)$ defined by $g(n):=\frac{4 n \sqrt{12 n^{2}-1}}{16 n^{2}-1}$ also approaches the value $\frac{\sqrt{3}}{2}$ as $n \rightarrow \infty$ remaining above this value. From there we have $\sin \alpha_{2}>\frac{\sqrt{3}}{2}$ and hence $\alpha_{2}>\frac{\pi}{3}$.

Finally, consider the triangle $S_{2 n} S_{2 n+1} S_{2 n+2}$. Its area is given by

$$
P_{2 n+1}=\frac{\sqrt{12 n^{2}+12 n+2}}{2 n(2 n+1)(2 n+2)},
$$

from where it follows

$$
\sin \alpha_{3}=\frac{4 n \sqrt{12 n^{2}+12 n+2}}{16 n^{2}+12 n+2} .
$$

Here, however, the function $h(n):=\frac{4 n \sqrt{12 n^{2}+12 n+2}}{16 n^{2}+12 n+2}$ approaches its limit $\frac{\sqrt{3}}{2}$ from below. Hence, $\alpha_{3}<\frac{\pi}{3}$, and we cannot conclude that the sum of three angles in the center of an even-numbered circle exceeds $\pi$.

The situation can be saved, however, if we manage to show that a sum of $\alpha_{3}$ and one of the remaining angles is at least $\frac{2 \pi}{3}$. It will follow if we prove, for example, $\arcsin f(n)+\arcsin h(n)>\frac{2 \pi}{3}$. By starting from

$$
\arcsin f(n)+\arcsin h(n)=\pi-\arcsin \left[f(n) \sqrt{1-h(n)^{2}}+h(n) \sqrt{1-f(n)^{2}}\right]
$$


and plugging in the expressions for $f(n)$ and $h(n)$ we obtain that $f(n) \sqrt{1-h(n)^{2}}+$ $h(n) \sqrt{1-f(n)^{2}}$ remains below $\frac{\sqrt{3}}{2}$ and tends increasingly toward this value. As this is the argument of the arcsin function on the right-hand side of the above expression, it means that the value of this arcsin function is below $\pi / 3$, and then the whole right-hand side exceeds the value of $\frac{2 \pi}{3}$, as desired.

Now we can establish the main result of this subsection.

Theorem 7 The sequence of circle centers for the packing with $a_{n}=n$ diverges.

Proof. Consider the piecewise linear curve $L=S_{2} S_{4} S_{6} \ldots$ It is a graph of a continuous function $\varphi(x)$ defined on some interval starting at $p_{2}$, the abscissa of $S_{2}$. The length of $L$ is then given by

$$
l(L)=\int_{p_{2}}^{M} \sqrt{1+\varphi^{\prime}(x)^{2}} d x
$$

where the integral is taken over the domain of the function $\varphi(x)$. Since the integrand is bounded on the whole domain, it follows that the infinite length of $L$ can be achieved only if $M=\infty$.

Corollary 8 Let $\left(a_{n}\right)$ be an admissible sequence with $a_{n} \in O(n)$. Then $\rho\left(\left(a_{n}\right)\right)=$ $\infty$.

Hence we have established divergence of circle centers for all packings with curvatures growing slower than the sequence of natural numbers. This, together with Theorem 7 and Corollary 3, settles the problem of convergence. Now it remains to look at convergent cases and see what we can say about their limits.

\section{$4.2 a_{n}=q^{n}$}

We have observed in section 3 that the abscissas of centers in the packing of circles whose curvatures are given by a geometric sequence appear in pairs. That means 
that the segments connecting the centers of circles whose indices have the opposite parity are vertical. This is not accidental; it is a consequence of the fact that for a geometric sequence of curvatures all triangles $T_{n}$ are similar, as it is shown in Figure 12. The proof is straightforward and we omit it.

A direct consequence of similarity of all $T_{n}$ is that the centers of all circles with

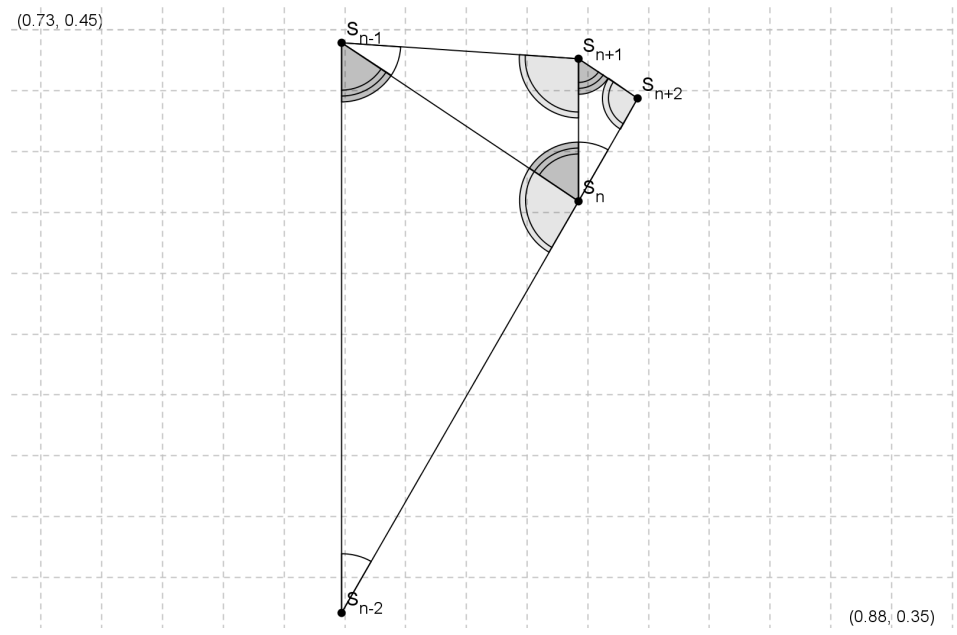

Figure 12: Triangle mesh for $a_{n}=q^{n}$

indices of the same parity lie on a line. From there, it follows that the sum of all lengths of segments connecting the center of even-numbered circles is actually the distance between $S_{0}$ and the limit. Since the same conclusion is valid for the sum of distances between the centers of odd-numbered circles, the limit must lie on the intersection of two circles with known centers and known radii. Hence, we have exact and explicit expressions for the coordinates of the limit $S\left(\left(q^{n}\right)\right)$. The proof is straightforward and we omit it.

Theorem 9 Let $\left(a_{n}\right)$ be a geometric sequence with quotient $q>1, a_{n}=q^{n}$. Then

$$
S\left(\left(q^{n}\right)\right)=\left(\frac{2 \sqrt{q+q^{2}+q^{3}}}{(q+1)\left(q^{2}-1\right)}, \frac{2 q}{(q+1)\left(q^{2}-1\right)}\right) \quad \text { and } \quad \rho\left(\left(q^{n}\right)\right)=\frac{2 \sqrt{q}}{q^{2}-1} .
$$


For our example $a_{n}=2^{n}$ this yields $S\left(\left(2^{n}\right)\right)=\left(\frac{2 \sqrt{14}}{9}, \frac{4}{9}\right)$ and $\rho\left(\left(2^{n}\right)\right)=\frac{2 \sqrt{2}}{3}$.

We have shown that a geometric sequence of curvatures forces similarity of triangles $T_{n}$. It can be shown that also the opposite is valid, hence that all $T_{n}$ are similar if and only if the sequence of curvatures is geometric. It is unclear whether this means that the geometric sequences are the only ones allowing exact solutions for the limit.

\section{$5 \quad$ Estimates for convergent sequences}

We know from section 2 that the sequence of circle centers converges for all curvature sequences $\left(a_{n}\right)$ such that $\sum \frac{1}{a_{n}}$ converges. In this section we present a method to estimate the position of the limit $S\left(\left(a_{n}\right)\right)$.

We start by finding the centers $S_{1}$ and $S_{2}$. Then we compute two sums. The first one is the total length of all segments between the centers of successive odd-numbered circles; the second one is the total length of all segments connecting the centers of successive even-numbered circles. Since $\sum \frac{1}{a_{n}}$ converges, both sums must be finite. Formally,

$$
R_{1}=\sum_{k=1}^{\infty}\left[\frac{1}{a_{2 k-1}}+\frac{1}{a_{2 k+1}}\right], \quad R_{2}=\sum_{k=1}^{\infty}\left[\frac{1}{a_{2 k}}+\frac{1}{a_{2 k+2}}\right] .
$$

In general case, $R_{1}$ and $R_{2}$ are lengths of piecewise linear curves connecting $S_{1}$ and $S_{2}$, respectively, with $S\left(\left(a_{n}\right)\right)$. Hence they can serve as estimates of the distances from $S_{1}$ and $S_{2}$, respectively, to $S\left(\left(a_{n}\right)\right)$. It means that $S\left(\left(a_{n}\right)\right)$ must be closer to both $S_{1}$ and $S_{2}$ than the point $S^{\prime}$ at the intersection of circles centered at $S_{1}$ and $S_{2}$ with radii $R_{1}$ and $R_{2}$, respectively.

We illustrate the quality of those estimates on two examples.

Example $10 a_{n}=n^{2}$. 
It follows immediately from the well known expression $\sum_{n=1}^{\infty} \frac{1}{n^{2}}=\frac{\pi^{2}}{6}$ that the expressions for sums of reciprocal values of squares of even and odd numbers are given by

$$
\sum_{n=0}^{\infty} \frac{1}{(2 n+1)^{2}}=\frac{\pi^{2}}{8}, \quad \sum_{n=1}^{\infty} \frac{1}{(2 n)^{2}}=\frac{\pi^{2}}{24} .
$$

From there we readily obtain

$$
R_{1}=2 \cdot \frac{\pi^{2}}{8}-1 \approx 1.4674011, \quad R_{2}=2 \cdot \frac{\pi^{2}}{24}-\frac{1}{4} \approx 0.572467
$$

By finding intersections of the circle centered at $(0,1)$ of radius $R_{1}$ with the circle centered at $\left(1, \frac{1}{4}\right)$ of radius $R_{2}$, we obtain two points. The one with both coordinates positive, $S^{\prime}=(1.4218,0.6370)$, can serve as an estimate of $S\left(\left(n^{2}\right)\right)$. We see from Fig. 13 that the estimate is not very good. This is a consequence of the fact that the piecewise linear curves connecting $S_{1}$ and $S_{2}$ to $S\left(\left(n^{2}\right)\right)$ are quite far from straight segments, leading thus to overestimates of the corresponding distances.

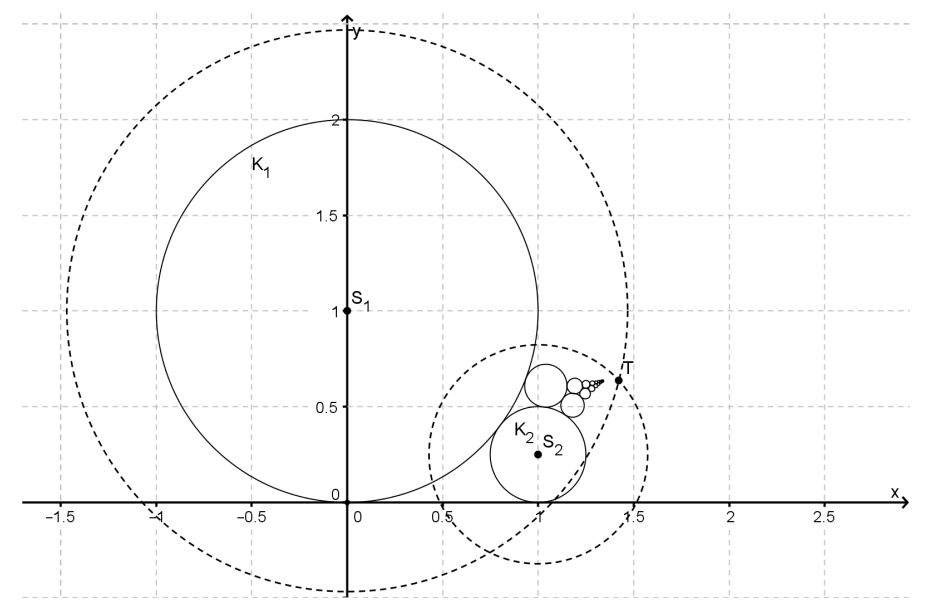

Figure 13: Estimate for $a_{n}=n^{2}$

Example 11 Our last example is concerned with Fibonacci numbers, $a_{n}=F_{n}$. 
There are explicit expressions for sums of the reciprocal values of odd- and evenindexed Fibonacci numbers. For example,

$$
\begin{gathered}
\sum_{n=0}^{\infty} \frac{1}{F_{2 n+1}}=\frac{1}{4} \sqrt{5} \vartheta_{2}\left(0, \frac{3-\sqrt{5}}{2}\right)^{2} \approx 1.824515, \\
\sum_{n=1}^{\infty} \frac{1}{F_{2 n}}=\frac{\sqrt{5}\left(2 \psi_{\phi^{-4}}^{(0)}(1)-4 \psi_{\phi^{-2}}^{(0)}(1)+\ln 5\right)}{8 \ln \frac{1+\sqrt{5}}{2}} \approx 1.535371 .
\end{gathered}
$$

(Here $\vartheta$ and $\psi$ denote the theta and digamma functions, respectively, and $\phi$ is the golden ratio [5]. However, we are interested mostly in the approximate values.) Starting from the above expressions we can derive the approximate values $R_{1}=$ 2.64903 and $R_{2}=2.070742$ for the radii of circles centered at $(0,1)$ and $(2,1)$, respectively. Their intersection with coordinates $S^{\prime}=(1.68235,3.04623)$ yields a pretty accurate estimate of the limit $S\left(\left(F_{n}\right)\right)$. We do not have an exact expression, but the value of $S_{20}=(1.67851,3.04503)$ given by our algorithm is both close to $S^{\prime}$ and closer to $S_{1}$ and $S_{2}$ than $S^{\prime}$ is to either of them, consistent with the fact that $S^{\prime}$ is an overestimate. Figures 14 and 15 show the quality of the estimate. We can

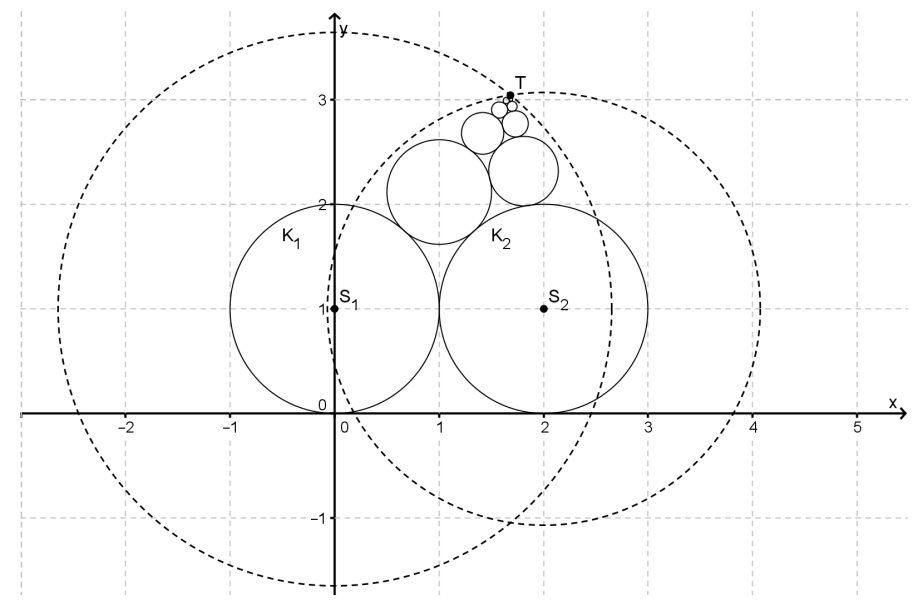

Figure 14: Estimate for $a_{n}=F_{n}$

observe that the estimate for Fibonacci numbers is much better than for the squares of natural numbers. The explanation is that the Fibonacci numbers asymptotically 


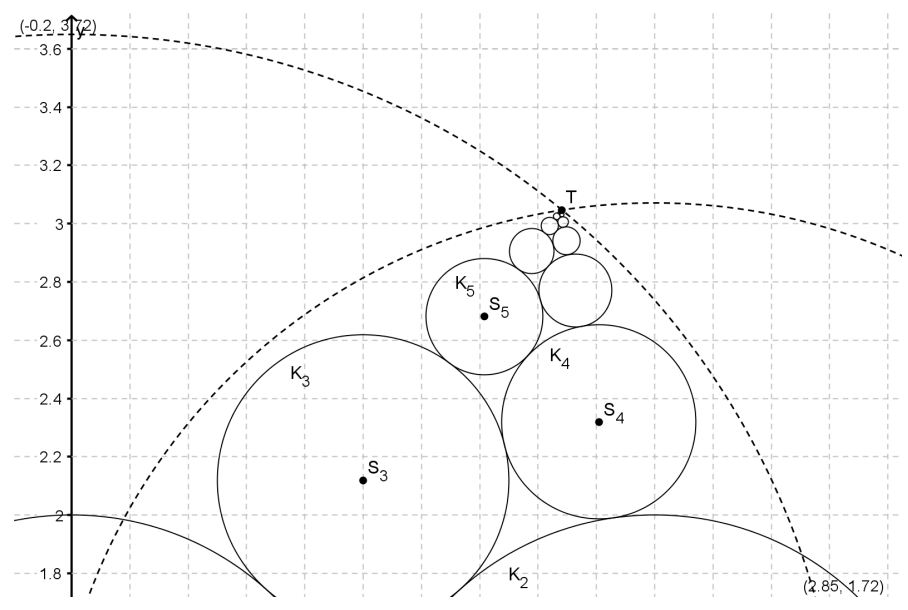

Figure 15: Zoom for $a_{n}=F_{n}$

behave as $F_{n} \sim \phi^{n}$ for large $n$. Hence they behave almost like geometric sequences, and for geometric sequences we have exact solutions.

\section{Concluding remarks}

In this paper we have analyzed behavior of directed packings of circles in the plane when their curvatures are given by a non-decreasing sequence of non-negative real numbers. We have established that the sequence of circle centers converges if and only if the growth rate of the sequence of curvatures strictly exceeds the growth rate of the sequence of natural numbers. For the case of geometric sequences $a_{n}=q^{n}$, $q>1$, we obtained exact coordinates of the limit $S\left(\left(q^{n}\right)\right)$, while in some other convergent cases we obtained good quality upper bounds on its distance from the origin.

It would be interesting to investigate if some other classes of sequences also allow explicit expressions for coordinates of their limits. Another interesting thing to do would be to consider three- (and maybe even higher-) dimensional analogues. We 
believe that at least in the three-dimensional case it should be possible to obtain some exact results.

\section{References}

[1] L. Fejes Toth, Lagerungen in der Ebene auf der Kugel und im Raum, SpringerVerlag, Berlin, Heidelberg, New York, 1972.

[2] T. C. Hales, S. P. Ferguson, The Kepler Conjecture: The Hales-Ferguson Proof, New York, 2011.

[3] T. Hales, M. Adams, G. Bauer, T. D. Dang, J. Harrison, L. T. Hoang, C. Kaliszyk, V. Magron, S. McLaughlin, T. T. Nguyen, Q. T. Nguyen, T. Nipkow, S. Obua, J. Pleso, J. Rute, A. Solovyev, T. H. A. Ta, N. T. Tran, T. D. Trieu, J. Urban, K. Vu, R. Zumkeller, A Formal Proof of the Kepler Conjecture, Forum of Mathematics, Pi 5: e2. doi:10.1017/fmp.2017.1

[4] The On-Line Encyclopedia of Integer Sequences, published electronically at https://oeis.org

[5] Wolfram Alpha, https://www.wolframalpha.com/ 


\section{Appendix}

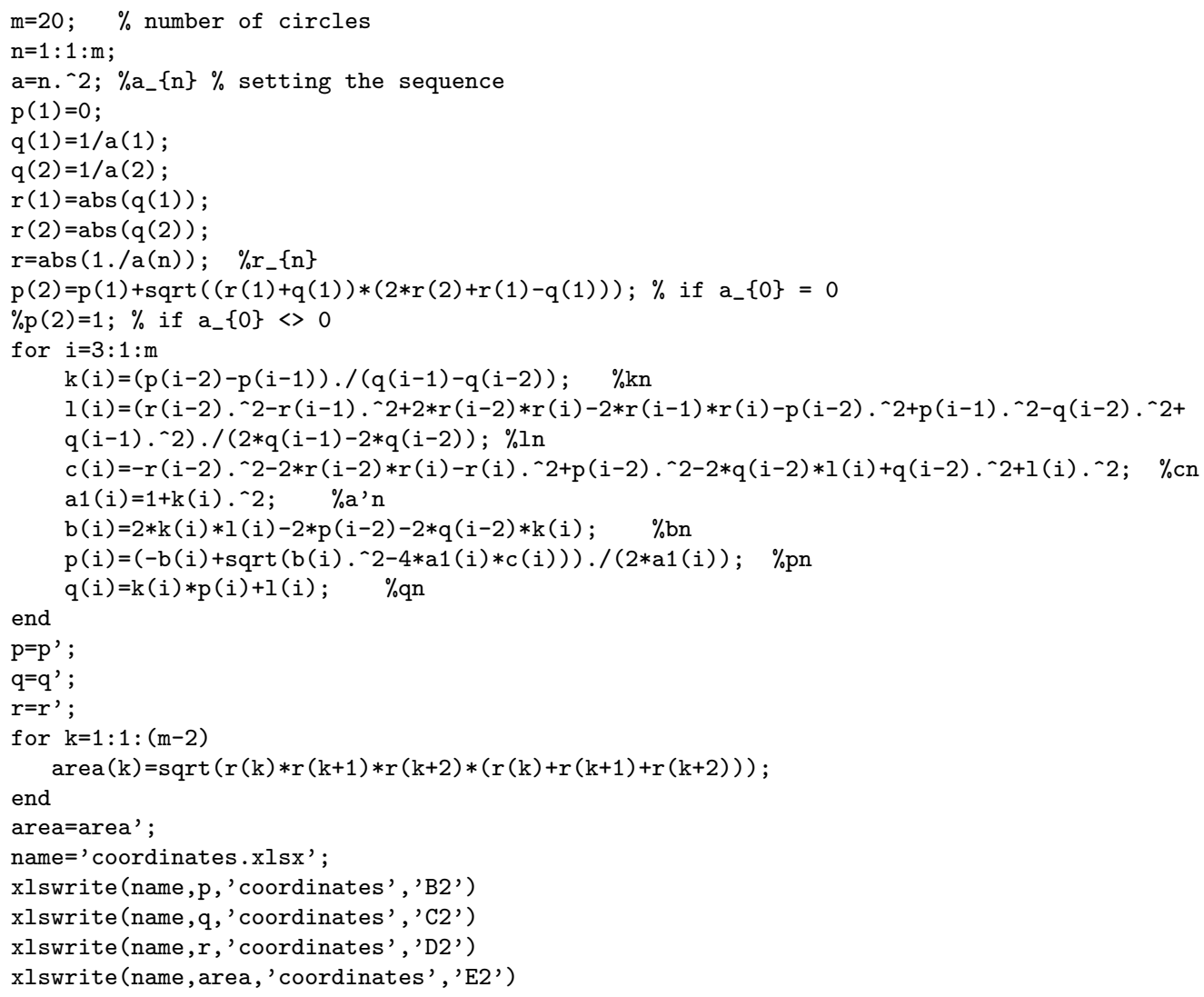

